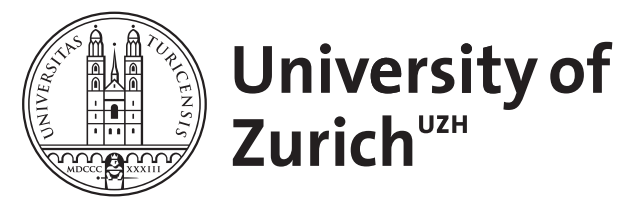

\title{
Abklärung von Kopfschmerzen
}

Schneider, Dominik

\begin{abstract}
Kopfschmerz ist eines der häufigsten medizinischen Probleme. Die lebenslange Prävalenz beträgt $94 \%$ bei Männern und $99 \%$ bei Frauen. In den USA sind Kopfschmerzen für 1-4\% aller Konsultationen auf einer Notfallstation verantwortlich [1] und damit der fünfthäufigste Grund [2,3]. 12-16\% der Menschen in Industrienationen leiden an Migräne, beim Spannungskopfschmerz liegt der Prozentanteil noch höher. Von den meisten primären Kopfschmerzformen sind Frauen häufiger betroffen, alsMänner, die Ausnahme stellt der Cluster-Kopfschmerz dar [4,5]. Neben dem subjektiven Leidensdruck, der durch Kopfschmerzen entsteht, sind auch die gesamtvolkswirtschaftlichen Kosten durch Behandlung, Abklärungen sowie Arbeitsausfälle von relevanter Bedeutung.
\end{abstract}

DOI: https://doi.org/10.1024/1661-8157/a001019

Posted at the Zurich Open Repository and Archive, University of Zurich ZORA URL: https://doi.org/10.5167/uzh-71677

Journal Article

Accepted Version

Originally published at:

Schneider, Dominik (2012). Abklärung von Kopfschmerzen. Praxis, 101:1001-1009.

DOI: https://doi.org/10.1024/1661-8157/a001019 


\section{Abklärung von Kopfschmerzen}

Kopfschmerz ist eines der häufigsten medizinischen Probleme. Die lebenslange Prävalenz beträgt $94 \%$ bei Männern und 99\% bei Frauen. In den USA sind Kopfschmerzen für 1-4\% aller Konsultationen auf einer Notfallstation verantwortlich [1] und damit der fünfthäufigste Grund [2,3].

12-16\% der Menschen in Industrienationen leiden an Migräne, beim Spannungskopfschmerz liegt der Prozentanteil noch höher. Von den meisten primären Kopfschmerzformen sind Frauen häufiger betroffen, als Männer, die Ausnahme stellt der Cluster-Kopfschmerz dar [4,5]. Neben dem subjektiven Leidensdruck, der durch Kopfschmerzen entsteht, sind auch die gesamtvolkswirtschaftlichen Kosten durch Behandlung, Abklärungen sowie Arbeitsausfälle von relevanter Bedeutung.

Für die meisten dieser Patienten ist der Hausarzt die primäre Anlaufstelle, der sie adäquat versorgen kann. Nur Wenige benötigen einen Neurologen oder gar eine spezialisierte Kopfschmerzsprechstunde.

Zur Differenzierung der verschiedenen Formen von Kopfschmerzen hat die International Headache Society (IHS) 1988 erstmals eine Klassifikation mit entsprechenden Diagnosekriterien ausgearbeitet. Diese wurde zuletzt 2004 überarbeitet und kann auf der Homepage der IHS studiert werden (Tab. 1).

Es wird in primäre und sekundäre Kopfschmerzen unterteilt. Bei Letzteren sind die Kopfschmerzen das Symptom einer zugrunde liegenden Erkrankung anderer Genese.

\section{Primärer Kopfschmerz}

1. Migräne

2. Kopfschmerz vom Spannungstyp

3. Clusterkopfschmerz oder andere trigemino-autonome Kopfschmerzen

4. Andere primäre Kopfschmerzen: Hustenkopfschmerz, Kopfschmerz bei körperlicher Anstrengung, Kopfschmerz bei sexueller Aktivität

Tab. 1:

Einteilung der Kopfschmerzformen nach IHS

\section{Sekundärer Kopfschmerz}

5. Kopfschmerzen zurückzuführen auf ein Kopf- oder HWS-Trauma

6. Kopfschmerzen zurückzuführen auf Gefässstörungen im Bereich des Kopfes oder des Halses: Ischämischer Hirninfarkt, intrakranielle Blutung, nicht-rupturierte Gefässanomalie, Arteriitis, Hirnvenenthrombose

7. Kopfschmerzen zurückzuführen auf nicht-vaskuläre intrakranielle Störungen:Liquordrucksteigerung, Liquorunterdruck, nicht-infektiöse entzündliche Erkrankungen, intrakranielle Neoplasmen

8. Kopfschmerzen zurückzuführen auf eine Substanz oder deren Entzug:Medikamentenübergebrauch, medikamentöse Nebenwirkung

9. Kopfschmerzen zurückzuführen auf eine Infektion

10. Kopfschmerzen zurückzuführen auf eine Störung der Homöostase: Arterielle Hypertonie, Dialyse

11. Kopf- oder Gesichtsschmerz zurückzuführen auf Erkrankungen des Schädels sowie von Hals, Augen, Ohren, Nase, Nebenhöhlen, Zähnen, Mund oder anderen Strukturen

12. Kopfschmerzen zurückzuführen auf psychiatrische Störungen

Kraniale Neuralgien, zentraler und primärer Gesichtsschmerz und andere Kopfschmerzen

13. Kraniale Neuralgien und zentrale Ursachen von Gesichtsschmerz

14. Andere Kopfschmerzen, kraniale Neuralgien, zentrale oder primäre Gesichtsschmerzen 


\section{Im Artikel verwendete Abkürzungen}

$\begin{array}{ll}\text { BSR } & \text { Blutsenkungsreaktion } \\ \text { CCT } & \text { Craniale Computertomographie } \\ \text { CRP } & \text { C-reaktives Protein } \\ \text { CT } & \text { Computertomographie } \\ \text { GCS } & \text { Glasgow Coma Scale } \\ \text { HWS } & \text { Halswirbelsäule } \\ \text { IHS } & \text { International Headache Society } \\ \text { KM } & \text { Kontrastmittel } \\ \text { LP } & \text { Lumbalpunktion } \\ \text { MRI } & \text { Magnetresonsztomographie } \\ \text { SAB } & \text { Subarachnoidalblutung }\end{array}$

U

Entscheidend auf dem Weg zu einer adäquaten Therapie ist eine korrekte Diagnose. Eine ausführliche und präzise Anamnese ist dabei unerlässlich und hilft unnötige apparative Abklärungen zu vermeiden [6]. Dabei muss insbesondere die Charakteristik des Kopfschmerzes, der zeitliche Verlauf, die Lokalisation, Begleitsymptome, Trigger sowie die persönliche Anamnese mit Nebenerkrankungen beachtet werden.

Aus der Anamnese ergeben sich häufig erste Weichenstellungen für die weiteren Abklärungen. Ein symptomatischer/sekundärer Kopfschmerz wird meist als ungewohnt bezüglich Charakter oder Stärke beschrieben. Mögliche Begleitsymptome sind dem Patienten bis anhin nicht bekannt. Der Beginn kann richtungsweisend sein. So ist beispielsweise ein perakutes Auftreten verdächtig auf eine Subarachnoidalblutung. Wenn die Beschwerden in spezifischen Situationen ausgelöst werden, oder erstmals nach einem bestimmten Ereignis auftraten, deutet dies auf einen symptomatischen Kopfschmerz hin (z.B. nach Trauma oder Gefässdissektionen).

Während die meisten primären Kopfschmerzen maximal wenige Tage andauern können, muss bei einem Dauerschmerz an intrakranielle Pathologien oder eine Allgemeinerkrankung mit Kopfschmerz als Leitsymptom gedacht werden und sollte daher weiter abgeklärt werden.

Neben der Anamnese kommt der klinischen Untersuchung mit Schwerpunkt auf den neurologischen Status eine wichtige Bedeutung zu. Bei der Mehrzahl der Patienten mit Kopfschmerz als Leitsymptom werden diese Untersuchungen unauffällig ausfallen. Umso wegführender und relevanter können daher pathologische Befunde wie z.B. Meningismus, fokal neurologische Defizite oder Papillenödem sein. Neben den Vitalparametern als allgemeine Orientierung muss die Temperatur als Hinweis für Infekte gemessen werden. Die Nackenmuskulatur muss ebenso wie die Wirbelsäule zur Beurteilung der muskuloskelettalen Situation untersucht werden. Strömungsgeräusche im Nacken-/Kopfbereich können auf vaskuläre Malformationen hindeuten. Die Region der Temporalarterie sollte auf Druckdolenz und Konsistenz hin beurteilt werden. 

Differenzialdiagnostische Überlegungen ((Ü1))

Die sekundären Kopfschmerzen, die potenziell gefährlich sein können, machen höchstens 10-20\% der Kopfschmerzen aus. Mit Abstand am häufigsten treten die primären Kopfschmerzen auf, von denen über $90 \%$ Migräne, Spannungs- oder Clusterkopfschmerzen sind. Somit kann bereits ein grosser Teil der Patienten durch die Differenzierung dieser Kopfschmerzarten diagnostiziert werden (Tab. 2). Dennoch bleiben viele diagnostische Unsicherheiten, da einerseits die Symptome unspezifisch sind und häufig bei mehreren Kopfschmerzformen vorkommen können und es andererseits mehrere auslösende Ursachen der Kopfschmerzen gleichzeitig geben kann.

Klinisch relevant ist in erster Linie die Identifizierung von potenziell bedrohlichen sekundären

Kopfschmerzen. Bei dieser ersten Einschätzung ist vor allem nach «Red flags» zu suchen (Tab. 3), die Anlass zu einer erweiterten apparativen Diagnostik sein sollten.

Anhand der bis hierhin erhobenen Befunde und gewonnenen Informationen inklusive der Warnzeichen kann eine erste

Risikoeinschätzung stattfinden. Als besonders aussagekräftig bezüglich relevanter zugrunde liegender Pathologien haben sich drei Kriterien herausgestellt: Alter über 50 Jahre, plötzlicher Beginn, und neurologische Befunde. Bei einer Kombination dieser drei Kriterien konnte eine Sensitivität von 98,6\%

\begin{tabular}{|c|c|c|c|}
\hline Symptom & Migräne & $\begin{array}{l}\text { Kopfschmerz } \\
\text { vom } \\
\text { Spannungstyp }\end{array}$ & Clusterkopfschmerz \\
\hline Lokalisation & $\begin{array}{l}\text { 70\% Unilateral } \\
30 \% \text { Bilateral }\end{array}$ & Bilateral & $\begin{array}{l}\text { Immer unilateral, } \\
\text { meist orbital, } \\
\text { supraorbital oder } \\
\text { temporal }\end{array}$ \\
\hline Charakteristika & $\begin{array}{l}\text { Crescendo Muster, } \\
\text { pulsierend, } \\
\text { Intensität moderat } \\
\text { bis stark, } \\
\text { intensiviert durch } \\
\text { physische Aktivität }\end{array}$ & $\begin{array}{l}\text { Drückend, } \\
\text { beengend, } \\
\text { Intensität leicht } \\
\text { bis moderat. } \\
\text { Keine } \\
\text { Intensivierung } \\
\text { durch } \\
\text { physische } \\
\text { Aktivität, kein } \\
\text { pulsierender } \\
\text { Charakter }\end{array}$ & $\begin{array}{l}\text { Attackenartig, } \\
\text { Intensität stark. } \\
\text { Treten in Clustern auf } \\
\text { mit Phasen der } \\
\text { Remission. }\end{array}$ \\
\hline Zeitdauer & 4-72 Stunden & $\begin{array}{l}30 \text { min bis } 7 \\
\text { Tage } \\
\text { (sporadische } \\
\text { Form) } \\
>3 \text { Monate } \\
\text { (chronische } \\
\text { Form) }\end{array}$ & $\begin{array}{l}15 \text { min bis } 3 \mathrm{~h} \text {, bis zu } \\
8 \text { Attacken pro Tag, } \\
\text { oft auch nachts. }\end{array}$ \\
\hline Begleitsymptome & $\begin{array}{l}\text { Teilweise mit } \\
\text { vorausgehender } \\
\text { Aura } \\
\text { Nausea, } \\
\text { Erbrechen, Phono- } \\
\text { /Photophobie }\end{array}$ & Keine & $\begin{array}{l}\text { Konjunktivale } \\
\text { Injektion, } \\
\text { Lakrimation, nasale } \\
\text { Kongestion, } \\
\text { Rhinorrhö, } \\
\text { Schwitzen, Miose, } \\
\text { Ptose, Agitiertheit, } \\
\text { Lidödem. }\end{array}$ \\
\hline $\begin{array}{l}\text { Verhaltensweise } \\
\text { des Patienten }\end{array}$ & Rückzugsverhalten & Unspezifisch & Agitiert, unruhig \\
\hline
\end{tabular}

Tab. 2: Anamnestische Hinweise zur Differenzierung der wichtigsten primären Kopfschmerzformen 
für einen sekundären Kopfschmerz nachgewiesen werden [7]. Die Intensität der Kopfschmerzen ist hingegen kein prädiktiver Faktor.

Tab. 3: «Red flags» der Kopfschmerzen, sortiert nach anamnestischen und klinischen Zeichen

- Erstmalige Kopfschmerzen, bzw. bisher unbekannte Kopfschmerzen

- Akuter, heftigster Kopfschmerz «wie noch nie» («thunderclap headache»)

- Zunahme der Symptomatik in Intensität oder Frequenz

- Dauerkopfschmerz

- Klar lokalisierter Kopfschmerz

- Epileptische Anfälle

- Vorgängiger Infekt

- Adäquates Trauma

- Orale Antikoagulation, Gerinnungsstörung

- Neoplasie in der persönlichen Anamnese

- Immunsuppression

- Fieber

- Fokal neurologische Ausfälle

- Vigilanzstörung

- Meningismus

- Neu aufgetretene neuropsychologische

Defizite

- Hirndruckzeichen
Basierend auf dieser Einschätzung kann entweder direkt eine Therapie noch vor Abschluss der Diagnostik eingeleitet werden, was beispielsweise bei einem Verdacht auf eine bakterielle Meningitis essentiell ist, oder es kann auf weitere Untersuchungsresultate wie Laborbefunde, Bildgebungen oder eine Lumbalpunktion gewartet werden.

Sind sekundäre Kopfschmerzen aufgrund der Anamnese, klinischen Untersuchung und allfälligen apparativen Abklärungen ausgeschlossen, kann die Diagnose anhand der Diagnosekriterien der IHS (Tab. 1) präzisiert und eine adäquate Therapie eingeleitet werden. 


\section{Abklärungsstrategie}

Zusatzabklärungen (Labor, Bildgebung) richten sich nach der durch Anamnese und Untersuchung erzielten Verdachtsdiagnose bzw. der diagnostischen Sicherheit [8].

Primäre Kopfschmerzen sind nicht mit strukturellen makroskopisch sichtbaren Veränderungen des Gehirns verbunden, weshalb eine bildgebende Abklärung nicht indiziert ist wenn Anamnese und Befund typisch sind und keine „Red flags“ bestehen. Häufig ist die Abgrenzung zu sekundären Kopfschmerzen jedoch nicht eindeutig.

Mittels kranialer Computertomographie können bereits in den nativen Bildern wichtige und gefährliche Ursachen wie eine frische Subarachnoidalblutung (SAB), ausgedehnte Hirntumore und Metastasen oder die akute Liquorabflussbehinderung erkannt werden. Da die Untersuchung schnell verfügbar und einfach durchzuführen ist, wird häufig das native CCT als Erstuntersuchung empfohlen, was bei nicht eindeutigen Ergebnissen durch das MRI im Verlauf ergänzt werden soll [9]. Beide Bildgebungsverfahren können bei unauffälligem Neurostatus primär ohne Kontrastmittel durchgeführt werden, allerdings macht es im «Nicht-Notfall-Setting» Sinn, das MRI mit Gadolinium zu wählen, um unnötige Zweituntersuchungen zu vermeiden. Bei der Fragestellung nach intrakraniellen Raumforderungen ist für eine präzise Aussage immer eine Kontrastmittelgabe notwendig. Bei einigen Diagnosen, wie dem subduralem Empyem, Hirnabszess, Enzephalitis, Dissektion, ist das MRI dem CCT überlegen und sollte als primäre Diagnostik angestrebt werden.

Die Lumbalpunktion ist beim Verdacht auf Meningitis indiziert, wobei in diesem Fall die Diagnostik den Therapiebeginn nicht verzögern darf. Ebenso, wenn die Anamnese sehr suggestiv für eine SAB ist, sollte bei «normaler» Bildgebung eine Lumbalpunktion durchgeführt werden. Denn in den ersten 24 Stunden nach einer Subarachnoidalblutung ist die Computertomographie (nur) in 95\% der Fälle diagnostisch relevant, d.h. es kann somit jede 20. Diagnose verpasst werden. Zweitens sinkt die Sensitivität der CT mit zunehmendem Zeitintervall zur stattgehabten SAB; eine Woche nach dem Ereignis sind nur noch 50\% der CT pathologisch und damit diagnostisch [9].

Der hier dargestellte Abklärungsalgorithmus (Abb. 1) kann nur eine grobe Richtlinie sein, die vom jeweiligen Fall abhängig angepasst werden muss. 


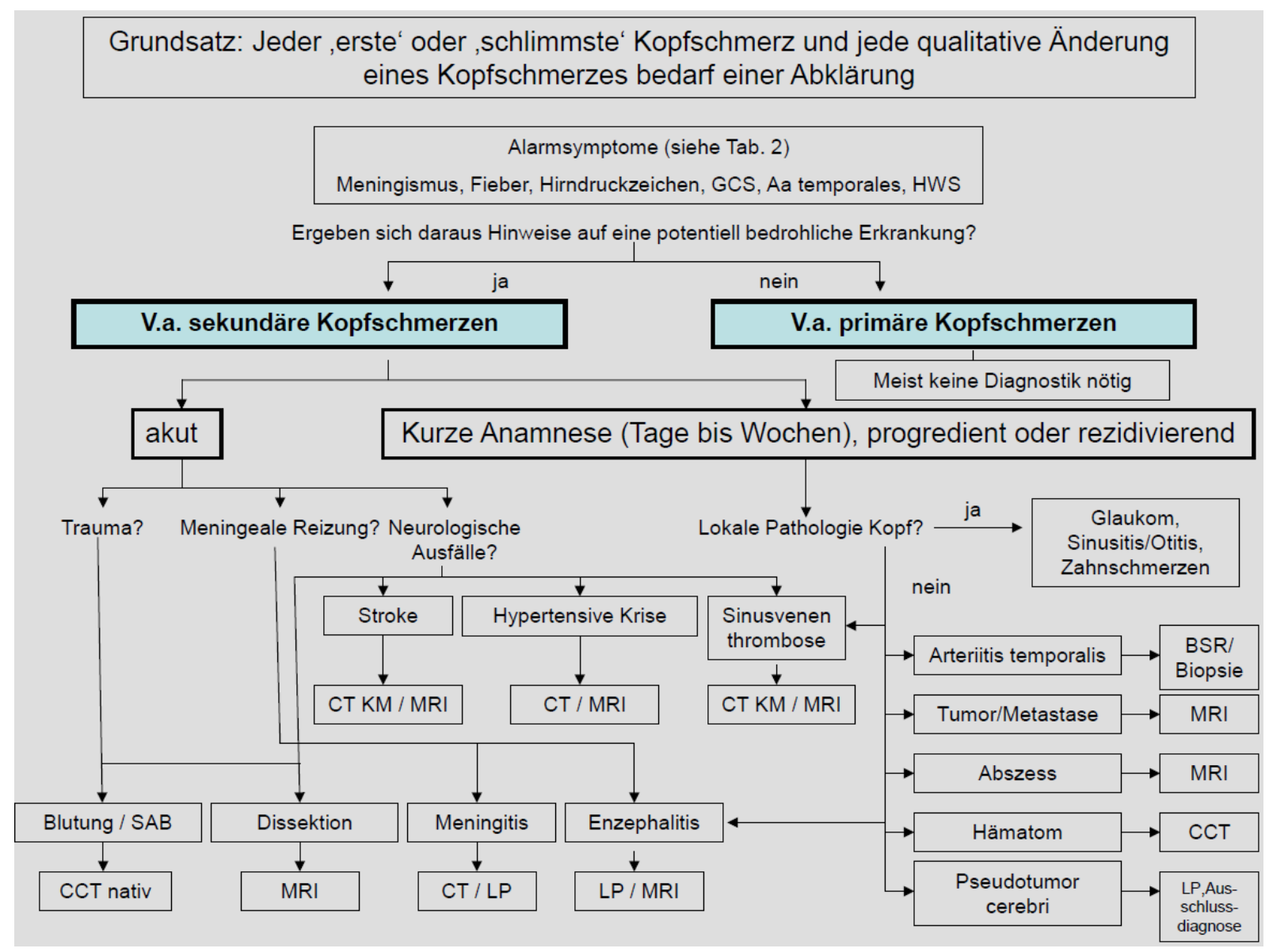

Abb. 1: Abklärungsalgorithmus (modifiziert nach Notfallstandards). Dies ist nur eine grobe Richtlinie, die Abklärungswege können im individuellen Fall abweichen. Abk.: BSR=Blutsenkungsreaktion, CCT=Craniale Computertomographie, CT=Computertomographie, GCS=Glasgow Coma Scale, HWS=Halswirbelsäule, $\mathrm{KM}=$ Kontrastmittel, LP=Lumbalpunktion, MRI=Magnetresonsztomographie, SAB=Subarachnoidalblutung

((Herr Kräuchi bitte folgende Änderungen in Abb.1: ((Titel)) «erste» «schlimmste»; ((3. Ebene)) potenziell; ((5. Ebene)) Akut; ((4. Ebene)) Ja, Nein)) 


\section{Fallbericht zur Abklärung von Kopfschmerzen}

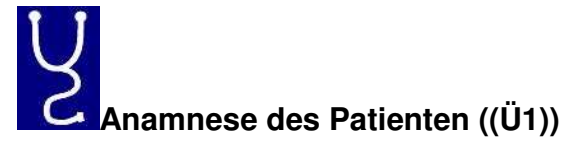

Eine 19-jährige Patientin meldet sich auf der Notfallstation wegen seit drei Wochen bestehenden starken

Kopfschmerzen. Diese sind von pochendem, pulsierendem Charakter, haben okzipital begonnen und sind nun holocephal mit Betonung frontal beidseits. Die Intensität ist fluktuierend, so dass die Patientin teilweise nachts wegen der Schmerzen erwacht und nicht mehr schlafen kann. Ein leichtes Ansprechen auf nicht-steroidale Antirheumatika ist vorhanden.

Ausserdem wird ein intermittierender, einige Sekunden anhaltender Visusverlust beschrieben.

Vier Tage vor Eintritt auf der Notfallstation suchte die Patientin eine Notfallpraxis auf, wo man aufgrund der Kopfschmerzen bei einem erhöhten CRP von 96 mg/l die Verdachtsdiagnose einer Sinusitis stellte und eine Antibiotikatherapie mit Amoxicillin/Clavulansäure einleitete, was jedoch nicht zu einer Linderung der Symptome führte. Im bisherigen Krankheitsverlauf hat die Patientin kein Fiebergefühl gehabt. Ein Trauma oder andere

Begleitsymptomatik werden bis auf eine leichte Nausea mit einmaligem Erbrechen vor vier Tagen verneint.

Eine Anamnese bezüglich vorbestehender Kopfschmerzen besteht nicht. Auch die übrige persönliche Anamnese ist unauffällig.

Noxen: Nikotinkonsum ca. 0,5 Packyear.

Die Familienanamnese war bezüglich Kopfschmerzen und insbesondere Migräne unauffällig.

Aktuelle Therapie: Orale Antikonzeption, Augmentin $1 \mathrm{~g}$ 2xtgl. seit vier Tagen, Mefenacid 500 mg 2xtgl. seit vier Tagen.

\section{Weg zur Diagnose/Kommentar ((Ü1))}

In der Erstbeurteilung dieser Patientin zeigen sich in der Anamnese rasch mehrere «Red flags», welche die Vermutung eines sekundären Kopfschmerzes und damit verbundene erweiterte Abklärungen nahelegen:

- Erstmalige stärkste Kopfschmerzen

- Dauerschmerz; nächtlicher Kopfschmerz

- Intermittierende neurologische Symptome (Visusverlust)

- Mögliche Hirndruckzeichen (Nausea, Erbrechen)

Für die Verdachtsdiagnose einer Sinusitis ergeben sich klinisch und anamnestisch wenige Hinweise (afebril, keine entsprechend lokalisierten Schmerzen, keine Rhinorrhö oder Verlegung der Nase). 


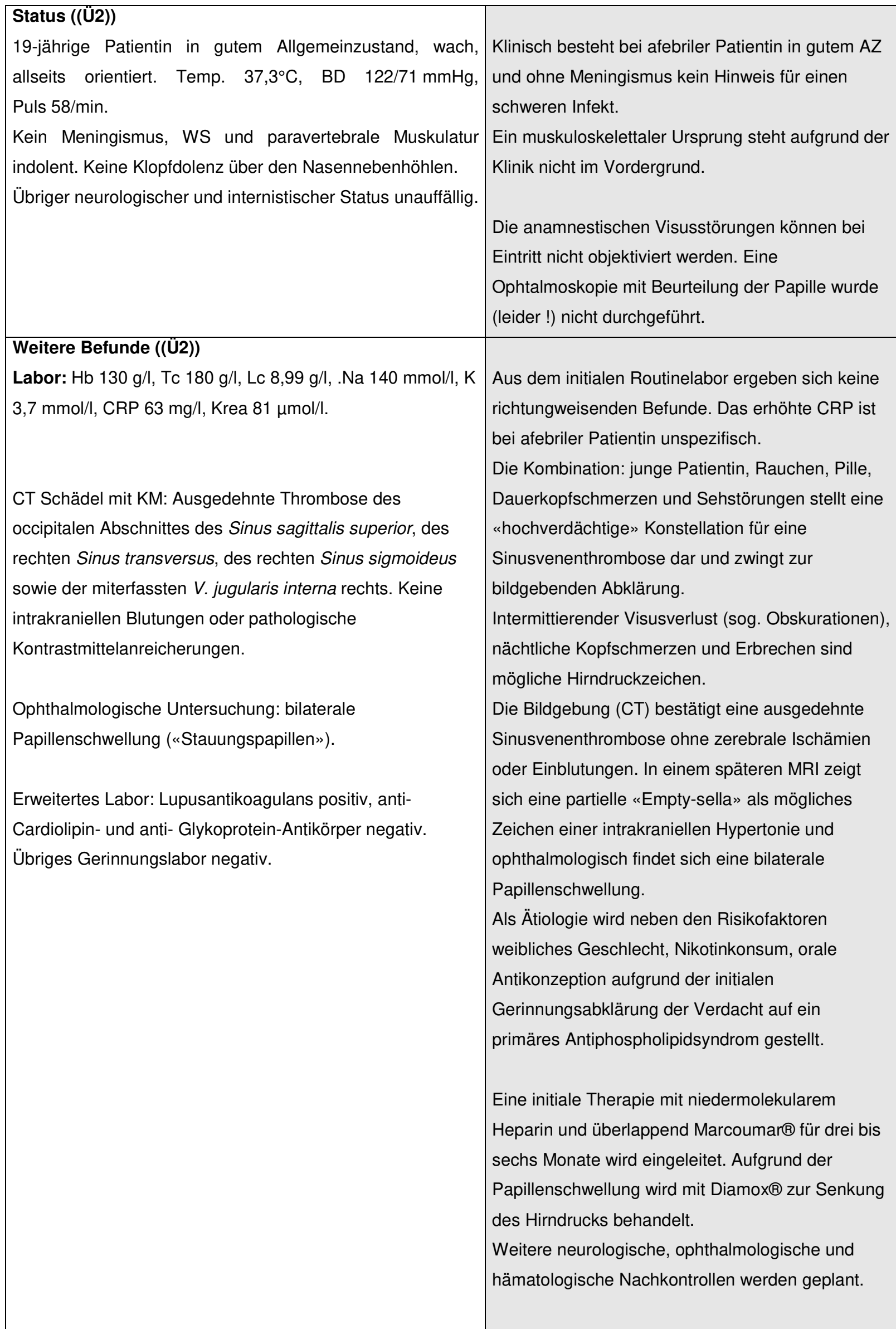




\section{Fragen zur Abklärung von Kopfschmerzen}

\section{Frage 1}

Welches der folgenden Symptome stellt keine «Red flag» dar? (Einfachauswahl, 1 richtige Antwort)
a) Fokales neurologisches Defizit
b) Erstmaliger bzw. bisher unbekannter Kopfschmerz
c) Erbrechen
d) Fieber

\section{Frage 2}

Welches ist die häufigste Kopfschmerzform? (Einfachauswahl, 1 richtige Antwort)
a) Migräne
b) Kopfschmerz vom Spannungstyp
c) Symptomatischer Kopfschmerz durch intrakranielle Raumforderungen
d) Medikamentenübergebrauchskopfschmerz (Müks)
e) Akute Subarachnoidalblutung

\section{Frage 3}

Welches Symptom/Aussage passt nicht zur Migräneattacke? (Mehrfachauswahl, mehrere richtige Antworten)
a) Die Zeitdauer der Migräneattacke reicht von 30 Minuten bis 7 Tage
b) Sie kann von Nausea, Erbrechen, Phono- oder Photophobie begleitet sein
c) Der Kopfschmerz ist in $70 \%$ der Fälle bilateral lokalisiert
d) Sehstörungen als Begleitsymptom
e) Einseitige konjunktivale Injektion als häufiges Begleitsymptom

\section{Frage 4}

Welche der folgenden Verdachtsdiagnosen kann eine Indikation zur Lumbalpunktion darstellen? (Mehrfachauswahl, mehrere richtige Antworten)
a) Bakterielle Meningitis
b) Virale Meningitis
c) Hirnabszess
d) Sinusvenenthrombose
e) Subarachnoidalblutung bei unauffälliger Computertomographie

\section{Frage 5}

Welche der folgenden Aussagen über Kopfschmerzen ist richtig? (Einfachauswahl, 1 richtige Antwort)
a) Bei der Mehrzahl der Patienten mit Kopfschmerzen als Leitsymptom findet sich im klinischen Status ein richtungsweisender pathologischer Befund.
b) Bei der Abklärung eines subduralen Empyems und eines Hirnabszesses ist die Computertomographie der Kernspintomographie überlegen.
c) Bei primären Kopfschmerzen findet sich kein morphologisches Korrelat, so dass eine zerebrale Bildgebung bei passender Anamnese und Klinik nicht indiziert ist.
d) Eine Immunsuppression stellt kein Alarmsymptom beim Leitsymptom Kopfschmerzen dar. 


\section{Korrespondenzadresse ((Ü2))}

Dr. med. Dominik Schneider

Klinik und Poliklinik für Innere Medizin

Universitätsspital

8091 Zürich

dominik.schneider@usz.ch

\section{Autoren}

Klinik und Poliklinik für Innere Medizin, Universitätsspital Zürich ${ }^{1}$; Universitätsklinik für Neurologie, Universitätsspital Bern, Inselspital, Bern ${ }^{2}$

${ }^{1}$ Dr. med. Dominik Schneider, ${ }^{2}$ Prof. Dr. med. Matthias Sturzenegger

\section{Bibliographie}

1. Grimaldi D, Nonino F, Cevoli S, Vandelli A, D’Amico R, Cortelli P: Risk stratification of nontraumatic headache in the emergency department. J Neurol 2009; 256: 51-57.

2. Barton CW: Evaluation and treatment of headache patients in the emergency department: a survey. Headache 1994; 34: 91-94.

3. Middleton K, Hing E: National hospital ambulatory medical survey: 2003 emergency department summary. Adv Data 2005; 366:1-36.

4. Rasmussen BK: Epidemiology of migraine. Biomed Pharmacother 1995; 49: 452-455.

5. Rasmussen BK, Jensen R, Schroll M, Olesen J: Epidemiology of headache in a general population-a prevalence study. J Clin Epidemiol 1991; 44: 1147-1157.

6. Detsky ME, McDonald DR, Baerlocher MO, Tomlinson GA, McCrory DC, Booth CM: Does this patient with headache have migraine or need neuroimaging? JAMA 2006; 296: 1274-1283.

7. Locker TE, Thompson C, Rylance J, Mason SM: The utility of clinical features in patients presenting with nontraumatic headache: an investigation of adult patients attending an emergency department. Headache 2006; 46: 954-961.

8. Cortelli P, Cevoli S, Nonino F, Baronciani D, Magrini N, Re G: Evidence-based diagnosis of nontraumatic headache in the emergency department: a consensus statement on four clinical scenarios. Headache 2004; 44: 587-595.

9. Krings $T$ et al. Bildgebende Diagnostik bei der Abklärung des Kopfschmerzes. Deutsches Ärzteblatt 2004; 45: 3026-3034. 


\section{DOI 10.1024/1661-8157/a001020}

((PX 17_Rubrik CME-Antworten))

\section{Antworten zu den Fragen zur Abklärung von Kopfschmerzen aus PRAXIS Nr. 16}

\section{Frage 1}

\section{Antwort c) ist richtig.}

Erbrechen ist ein unspezifisches Symptom und stellt u.a. ein typisches autonomes Begleitsymptom bei einer Migräneattacke dar. Bei einem Patienten ohne Migräneanamnese und mit Dauerkopfschmerz kann aber Erbrechen, besonders Nüchternerbrechen ein Hirndruckzeichen darstellen.

Eine fokales neurologisches Defizit (anamnestisch z.B. halbseitige Sensibilitätsstörung oder Gesichtsfeldausfall oder bei der Untersuchung) ist eine «Red flag». Bei einem Patienten mit bekannter Migräne und immer wieder begleitender Aura z.B. mit halbseitigem Flimmerskotom und halbseitigen Parästhesien muss ein solches jedoch kein Alarmsyptom sein. Eine Aura dauert typischerweise weniger als 60 Minuten und zeigt einen sogenannten «march», d.h. eine Ausbreitung der (Reiz-)Symptome mit der Zeit (z.B. Wanderung der Flimmersensationen vom Zentrum in die Peripherie des Gesichtsfeldes oder der Parästhesien von den Fingern zur Hand und dann zum Mund stellt kein Warnsymptom dar.

\section{Frage 2}

\section{Antwort b)ist richtig.}

Primäre Kopfschmerzen machen 80-90\% aller KS-Formen aus. Während die Migräne (wegen KS-Intensität und der Begleitsymptome) zu den meisten Notfallkonsultationen führt, ist der Kopfschmerz vom Spannungstyp in der Gesamtbevölkerung die häufigste Kopfschmerzform.

\section{Frage 3}

Die Antworten a), c) und e) sind richtig.

Die Aussagen b) und d) sind typisch für eine Migräne.

Die typische Zeitdauer einer Migräneattacke liegt bei 4-72 Stunden. Die in Antwort a) angegebene Zeitdauer passt am ehesten zur sporadischen Form des Kopfschmerzes vom Spannungstyp.

Die Lokalisation der Kopfschmerzen bei Migräne sind in 70\% der Fälle unilateral bzw. unilateral betont, kann aber zwischen den einzelnen Attacken die Seite wechseln, oder sich auch innerhalb einer Attacke ausbreiten. Sehstörungen sind häufige Begleiterscheinungen bei Migräneattacken. Als Auraphänomen sind die Flimmerskotome häufig. Aber auch Patienten mit Migräne ohne Aura geben häufig weniger spezifische Sehstörungen (Verschwommensehen, Nebelsehen, Blendungsgefühl, Leseunfähgikeit) an.

Eine einseitige (ipsilateral zum streng einseitig und periokulär lokalisierten KS) konjunktivale Injektion ist ein typisches Begleitsymptom der Clusterkopfschmerzattacke; meistens mit zusätzlich enger Lidspalte und Augentränen sowie (ipislateralem) Nasenlaufen. Eine leichte eher bilaterale Rötung der Konjunktiven kann auch bei Migräne beobachtet werden.

\section{Frage 4}

Die Antworten a), b) und e) sind richtig.

Der Verdacht auf eine Meningitis bedingt eine umgehende Lumbalpunktion zwecks Diagnosesicherung (auch Abgrenzung zur SAB und bakteriell vs. viral) und Erregernachweis (Letzterer sollte aber immer auch aus dem Blut 
gemacht werden!). Bei Verdacht auf bakterielle Meningitis muss eine Antibiotikatherapie bereits vor der diagnostischen Abklärung gestartet werden. Der Hirnabszess ist eine mittels Bildgebung zu stellende Diagnose, der Liquorbefund kann sogar normal sein. Dasselbe gilt für die Sinusvenenthrombose.

Quantitativ geringgradige oder bereits vor einigen Tagen abgelaufene Subarachnoidalblutungen können der computertomographischen Erkennung entgehen. Ist z.B. ein Aneuryma die Ursache, so besteht dennoch ein hohes Rezidivrisiko, sodass die Diagnose bei hohem klinischem Verdacht mittels LP zu erzwingen ist (xanthochromer Liquor, Nachweis von Hämoglobinabbauprodukten oder von Erythrophagen)..

\section{Frage 5}

\section{Antwort c) ist richtig.}

Da die Patienten mit primären Kopfschmerz nur ausnahmsweise während einer Attacke untersucht werden können, ist der neurologische Untersuchungsbefund bei den meisten Kopfschmerz-Patienten unauffällig. Bei einem aufgrund von Anamnese, Verlauf und Untersuchungsbefund typischen primären Kopfschmerz ist eine Bildgebung nicht indiziert. Wohl aber, wenn (evtl .zusätzlich!) «Red flags» bestehen. Denn bei der hohen Lebenszeit-Prävalenz von primären Kopfschmerzen ist es sehr wohl möglich, dass z.B. eine Migränepatientin im Laufe ihrer «Kopfschmerz-Karriere» auch eine (zusätzliche) andere Kopfschmerz-Form z.B. eine Sinusvenenthrombose erleidet. Im klinischen Alltag ist zudem die zuverlässige Differenzierung zwischen primärem und sekundärem Kopfschmerz nicht immer möglich, so dass eine Bildgebung «aus Sicherheitsgründen» angebracht sein kann. 